\title{
CORRIGENDUM
}

\section{Spin-resolved quantum-dot resonance fluorescence}

A. Nick Vamivakas, Yong Zhao, Chao-Yang Lu and Mete Atatüre

Nature Physics 5, 198-202 (2009); published online: 25 January 2009; corrected after print: 1 December 2009.

The authors wish to point out that in all versions of this Letter originally published, the pure spontaneous emission rate of $356( \pm 11)$ $\mathrm{MHz}$ quoted on pages 198 and 199 is overestimated by a factor or four owing to an instrumentation-setting error, and erroneously quoted in units of angular frequency. The correct number should read $227( \pm 7) \mathrm{MHz}$ in linear frequency. The upper bound for fast dephasing mechanisms is therefore limited to $81 \mathrm{MHz}$, not $18 \mathrm{MHz}$. The main arguments of the paper are unaffected by this correction. These changes have been made in both PDF and HTML versions of this Letter. 